Dieses Dokument ist eine Zweitveröffentlichung (Verlagsversion) / This is a self-archiving document (published version):

Alexander Lasch, Wolf-Andreas Liebert

\title{
Sprache und Religion
}

Erstveröffentlichung in / First published in:

Ekkehard Felder/Andreas Gardt, Hrsg. Handbuch Sprache und Wissen. Berlin/Boston: De Gruyter 2015, S. 475 - 492. ISBN 978-3-11-029597-9.

DOI: https://doi.org/10.1515/9783110295979.475

Diese Version ist verfügbar / This version is available on:

https://nbn-resolving.org/urn:nbn:de:bsz:14-qucosa2-748496 


\title{
Alexander Lasch/Wolf-Andreas Liebert \\ 22. Sprache und Religion
}

\begin{abstract}
Der linguistische Forschungsstand zum Thema „Sprache und Religion“ weist im Vergleich zu anderen Disziplinen wie der Philosophie und Soziologie deutliche Lücken auf. Dies heißt jedoch nicht, dass das Thema keine Rolle spielte. Allerdings sind die bisherigen Untersuchungen zumeist sprachgeschichtlich ausgerichtet. Synchrone Untersuchungen sind erst in jüngster Zeit zu verzeichnen, insbesondere zu Ritualen, Kommunikationstypen und Textsorten. Studien zur Sprache anderer Religionen als dem Christentum, etwa zu populären Religionen oder den Bereichen von Spiritualität und Esoterik, wie sie beispielsweise in der Soziologie seit Jahren publiziert werden, stellen in der Linguistik ein Desideratum dar. Es wird daher zunächst eine Analyse des Feldes „Sprache und Religion“ durchgeführt, die als Ergebnis religiöse Sprache als funktionale Varietät ausmacht und wesentliche Elemente in Form der Schlüsselwörter Verkündigung, Verehrung, Vergegenwärtigung, Charisma, Unsagbarkeit, das Transzendente und Metaphysik identifiziert. Diese Schlüsselwörter stellen zentrale Lemmata eines Handbuchs „Sprache und Religion“ dar und werden im Einzelnen beschrieben. Schließlich werden methodische Überlegungen angestellt. Dabei werden zunächst die etablierten lexikologischen, lexikographischen, textlinguistischen und editionsphilologischen Verfahren angeführt, um schließlich auf die Potenziale ethnographischer, interaktionaler und diskurslinguistischer Methoden hinzuweisen.
\end{abstract}

1 Zum Stand der Forschung

2 Eine situative Analyse des Phänomenbereichs „Sprache und Religion“

3 Zentrale Lemmata

4 Methodische Überlegungen

5 Literatur

\section{Zum Stand der Forschung}

In der Linguistik ist das Thema „Sprache und Religion“ zunächst eine an der Textgeschichte der christlichen Religionen orientierte Forschung. Besonderes Interesse brachte man vor allem der Lutherbibel und den vielfältigen Wechselwirkungen zwischen Alltagssprache und Luthers Bibelübersetzung entgegen. Die Luther-Übersetzung wurde sogar aufgrund des prägenden Einflusses auf die deutsche Sprache als sprachgeschichtliche Zäsur begriffen und in einigen Ansätzen zur Begründung der Epoche des Frühneuhochdeutschen herangezogen. Ein weiterer Schwerpunkt ist, 
wie in den Literaturwissenschaften, die Auseinandersetzung mit der so genannten ,Mystik‘ (z. B. Stötzel 1966).

In dieser philologischen und auch lexikologischen und lexikographischen Tradition stehen die ersten Arbeiten, die das spezifische Thema Religion systematisch zunächst linguistisch (Moser 1964) und dann auch interdisziplinär fokussieren (Kaempfert 1971, 1983). Bereits diese frühen Arbeiten verlegen das Thema „Sprache und Religion“" aus dem Gegenstandsbereich einer linguistischen Textanalyse in den breiteren Interessenhorizont der Soziolinguistik. Insgesamt aber kann man den Umgang der Linguistik mit sprachlich-kommunikativen Phänomenen der Domäne „Religion“ durchaus als zögerlich charakterisieren. „Sprache und Religion“, das bedeutet in der Linguistik in erster Linie die Würdigung sprachhistorisch relevanter Texte und Textverbünde, wie etwa Bibelübersetzungen, Evangelienharmonien oder Zaubersprüche, wie sie für das Althochdeutsche und Altniederdeutsche typisch sind, religiös motivierte Dichtung und Traktatliteratur aus mittelhochdeutscher Zeit oder die Bedeutung der Schriften Martin Luthers für die Herausbildung eines überregionalen Standards im Frühneuhochdeutschen (vgl. dazu exemplarisch von Polenz 2000, 1994). Die religiösen Praxen, in die diese Zeugnisse eingebunden waren, spielten immer eine eher untergeordnete Rolle - Predigten wurden bspw. nicht als kommunikative Ereignisse der Verkündigung analysiert, sondern in der Form von Predigtsammlungen und -drucken. Hugo Mosers Arbeit Sprache und Religion (1964) und auch August Langens Wortschatz des deutschen Pietismus (1968) sind vor diesem Hintergrund zwar besonders herauszuheben als Studien, die sich dieses Themas annahmen, allerdings schlossen in den Folgejahren nur wenige Arbeiten an ihre Fragestellungen an. Während die so genannte pragmatische Wende innerhalb vieler Bereiche der Linguistik zu einer Neuausrichtung des Forschungsinteresses hin zur Sprache in ihrem Gebrauch und ihrer Rolle im kommunikativen Kontext führte, stagnierte die Entwicklung der Linguistik im Bereich „Sprache und Religion“. In anderen Disziplinen wie der Religionswissenschaft, der Ethnologie, der Soziologie und auch der Psychologie war dagegen eine große Dynamik zu verzeichnen, die bis heute ungebrochen ist.

Daher ist die gegenwärtige linguistische Forschung in großem Maße auf die Beiträge anderer Disziplinen angewiesen. Für die Linguistik erwies sich insbesondere die Perspektive der Ethnologie und der Soziologie als besonders fruchtbar. In der Ritualforschung wird etwa versucht, generelle Handlungsmuster des Rituellen zu erarbeiten, die sich auf die Analyse religiöser Praxen gründete. Einschlägig sind hier die Publikationen von Viktor Turner (z. B. 1989, 1967) und Hans Georg Soeffner (z. B. 1989). In der Religionssoziologie wurden in den letzten Jahren eine Fülle von Arbeiten zu unterschiedlichen sozialen und kommunikativen Dimensionen zeitgenössischer Religion und Spiritualität (vgl. etwa Knoblauch 1989, 2009, 1999, 2003; Gebhardt et al. 2005) vorgelegt.

Die Öffnung des Blicks auf Phänomene, die ihre kulturelle und gesellschaftliche Relevanz vor allem der Partizipation bzw. Adaptation religiöser und ritueller Praxen 
verdanken, brachte einige Studien hervor, die nicht mehr nur die in (meist christlichen) Glaubensgemeinschaften tradierten Textsorten und Exemplare derselben untersuchten (exemplarisch Werlen 1984, 1987; Paul 1990, 2009), sondern sich der Untersuchung von säkularen Ritualen zuwendeten, die typische Muster religiöser Rituale übernahmen (Fix 1998). Dennoch ist die Forschungslage auch heute noch recht überschaubar, um nicht $\mathrm{zu}$ sagen dürftig: Wenige Einzeluntersuchungen zu einzelnen Textsorten (Moser 1981; Greule 1992, 1999a und b, 2004; Funk 1991; Lasch 2005, 2013; Pfefferkorn 2005a und b; Grözinger 2009; Paul 2009; Klug 2012) oder zum Themenkomplex Rhetorik und Stilistik der ,religiösen Sprache“ (Fix 1998; Grözinger 2009; Paul 2009) bilden die Ausnahme. Auch typologische Überblicksdarstellungen sind selten und beziehen sich, ganz in der Tradition der sprachhistorischen Annäherung, meist auf die „Textsorten des religiösen und kirchlichen Bereichs“ (Simmler 2000, vgl. weiter 2005a, 2005b, 2007). Arbeiten, die sich der Frage nähern, in welchen kommunikativen Kontexten Sprache welche Rolle in religiösen Praxen spielt, sind leider selten (vgl. Lasch 2011). Gleiches gilt unverständlicherweise für Studien, die sich dem Zusammenhang von Text und Bild widmen (Klug 2012). In jüngster Zeit gibt es erste Ansätze in der multimodalen Gesprächsforschung, die bislang aber noch Mikroausschnitte fokussieren (Hausendorf/Schmitt, 2010; Schmitt 2012).

Sieht man von Hugo Mosers Ansatz (1964) ab, der religiös motivierten Sprachgebrauch als Sondersprache ansetzte, dann hat das Thema „Sprache der Religion“ als Variation der deutschen Sprache bzw. als Variationsgröße für Sprachen bisher wenig Beachtung gefunden, obwohl eine Einordnung des Themas in diesem Bereich am vielversprechendsten erscheint. Zwar wurde das Thema Religion insbesondere in der Kontaktlinguistik aufgegriffen, meist aber unter Gesichtspunkten wie der Erforschung von Sprachminderheiten oder Sprachkonflikten (vgl. Ammon et al. 2011). Wenn Religion als variierender Faktor angenommen wird, so könnte man zunächst an eine domänenspezifische Variation denken, wie sie etwa im Heidelberger Forschungsnetzwerk „Sprache und Wissen“ vorgenommen wird (Felder 2008, 2009). Bei genauerem Hinsehen fällt es allerdings auch auf den zweiten Blick nicht leicht zu bestimmen, was die Spezifik dieser Domäne nun genauer ausmacht. Kennzeichen des religiösen Bereichs sind sowohl eigene Gegenstände, ein Bezug zur Transzendenz als auch eine mehr oder weniger ausgearbeitete Metaphysik. Daneben sind Praktiken zu beobachten und $\mathrm{zu}$ beschreiben, die spezifische kulturelle Bedürfnisse und Zwecke synästhetisch erfüllen. Diese Beschreibung legt nahe, dass aus dieser Perspektive religiöse „Sprachen“ und die „Sprache der Religion“ systematisch als funktionale Varietät zu bestimmen ist. Dies ermöglicht es, z. B. Aspekte der Fachsprachenforschung auf die Beschreibung dieser Varietät zu übertragen: Sie schlägt bspw. eine horizontale und eine vertikale Differenzierung von verschiedenen funktionalen Varietäten vor (Roelcke 2010, 29 ff.). Während die horizontale Gliederung den verschiedenen Religionen auf inhaltlicher Ebene entsprechen würde, würde die vertikale Gliederung Graden auf einer Skala mit den Endpunkten maximaler interner und maximaler externer Kommunikation entsprechen. Das ist besonders attraktiv, um die 
verschiedenen Kommunikationsbereiche der „Sprache der Religion“ systematisch zu erfassen.

Nimmt man den Phänomenbereich „Sprache und Religion“ in den Blick, wählt man eine interdisziplinäre Perspektive auf Phänomene religiöser Kommunikation auf der einen Seite als auch auf Phänomene der Adaptation kommunikativer Muster religiöser Kommunikation auf der anderen Seite. Das Fehlen linguistischer Forschung zum Thema „Sprache und Religion“ in pragmatischer Hinsicht könnte nun für die Auseinandersetzung mit dem Thema zur Konsequenz haben, dass man den Phänomenbereich „Sprache und Religion“ ausschließlich oder vornehmlich durch Autoren nicht-linguistischer Provenienz umreißen lässt. Dies wäre aber für die Etablierung eines linguistischen Forschungsgegenstandes sehr unbefriedigend gewesen. Um den Gegenstand adäquat beschreiben zu können, sind zunächst die Ergebnisse der Nachbardisziplinen von höchster Relevanz, die die linguistischen Einzeluntersuchungen auf einem Feld einleiten, das mit einer Reihe von Leitbegriffen, die wir im Folgenden lemmatisieren, abgesteckt sei. Diese gewinnen wir aus einer situativen Analyse des Phänomenbereichs „Sprache und Religion“, ausgehend von der Prämisse, dass religiöse Sprache als eine funktionalen Varietät aufzufassen ist.

\section{Eine situative Analyse des Phänomenbereichs „Sprache und Religion“}

Auch wenn wir „Sprache und Religion“ linguistisch untersuchen, ist es gegenstandskonstitutiv, eine Größe für das im weitesten Sinne Transzendente anzusetzen. Dies soll aber keine Entscheidung über eine Existenz oder Nichtexistenz eines Numinosen bedeuten, sondern lediglich akzeptieren, dass innerhalb eines religiösen Bereiches von denjenigen, die zur Konstitution dieses Bereichs beitragen, ein wie auch immer geartetes Transzendentes als existierend angenommen wird.

Für die Erforschung eines solchen Bereiches, der immer auch ein Nicht-Sichtbares, Nicht-Greifbares als sichtbar und greifbar konstitutiv voraussetzt, ergeben sich eine Reihe von Konsequenzen, die ganz wesentlich aus den Eigenschaften dieses Transzendenten herrühren.

Zunächst einmal ist damit (analytisch) eine Zweiwelten-Struktur gegeben, wie wir sie aus der Literatur, etwa dem Märchen oder der phantastischen Literatur kennen: Die Unterscheidung zweier Welten ist insofern analytisch, als von den Teilnehmern einer religiösen Tradition durchaus keine Trennung erfahren werden muss. Allerdings ist das Transzendente nicht Teil der sinnlich wahrnehmbaren Welt, sondern wirkt in diese hinein. Daher stellt sich unmittelbar die Frage, in welcher Weise es mit Menschen, die nicht dem transzendenten Bereich angehören, in Verbindung treten und kommunizieren kann. 
Dieses Grundproblem einer kommunikativen Verbindung zwischen einem Transzendenten und einer Menschenwelt wird von den Religionen in höchst unterschiedlicher Weise angegangen und gelöst. Zum einen besteht die Möglichkeit, dass das Transzendente in Gestalten der menschlichen Welt verkörpert werden und direkt in der sinnlich wahrnehmbaren Welt auftreten, wirken und dann auch meist kommunizieren kann. Die Verkörperung des Transzendenten in der Menschenwelt ist dabei aber nur eine - wenngleich prominente - Möglichkeit, die Kommunikation zwischen den beiden Welten zu ermöglichen. Daneben kann das Transzendente in vielfältiger Weise mit Menschen kommunizieren, ohne körperlich in der Menschenwelt zu erscheinen. Meist stehen dann bestimmte Figuren im Mittelpunkt, die als Vermittler zwischen dem Transzendenten und der Menschenwelt fungieren und daher aus einer Gemeinschaft besonders herausgehoben sind: Sie können dabei Träger institutionalisierter (Papst, Pfarrer, Imam etc.) oder auch nicht-institutionalisierter Rollen (Religionsgründer, Propheten, Gurus) sein. Immer jedoch tragen sie das Charisma, nämlich das des Amtes oder das des unmittelbar vom Transzendenten erfüllten. Gerade die letzte Kategorie verweist auch auf eine Unterscheidung von William James (1997), nämlich die in eine institutionalisierte und eine persönliche Religion. Die persönliche Religion ist dabei ein auf das Transzendente gerichtetes Handeln, wodurch sich der Handelnde in einen unmittelbaren Austausch und in Kommunikation mit diesem Transzendenten begibt. Institutionalisierte Religion besitzt dagegen in der Regel eine Mittlerrolle zwischen Transzendentem und Anhängern. Die Mittlerrollen zielen darauf ab, den Anhängern einer bestimmten Religion spezifische Handlungsweisen qua Autorität des Transzendenten zu verkünden und damit auch normativ auf diese einzuwirken. Dies kann wie James gezeigt hat - durchaus auch auf die aus einer persönlichen Religion heraus begründeten Fälle von Religionsstiftern zutreffen, die damit auch eine Institutionalisierung erfahren. Eine andere Strategie besteht darin, die Kultur einer religiösen Erfahrung im Sinne einer unmittelbaren Begegnung mit dem Transzendenten zu pflegen. Religionen, die auf die Ermöglichung einer solchen persönlichen religiösen Erfahrung abzielen, wie etwa der Buddhismus, besitzen dementsprechend auch ein anderes Konzept des Vermittlers, dem eher die Rolle eines Mentors zukommt, der zur persönlichen religiösen Erfahrung anleitet und diese ermöglicht. So konzeptualisiert der Buddhismus diese Rolle beispielsweise als „Freund“ und nicht als „Pater“. Da als ein zentrales Merkmal zeitgenössischer Religiosität das verstärkte Bedürfnis nach dieser Form der persönlichen Religion ausgemacht werden kann (Gebhardt et al. 2005), stellt es auch einen Faktor dar, warum Religionen wie der Buddhismus in der Spätmoderne verstärkt Zulauf verzeichnen.

Eine weitere Besonderheit im religiösen Bereich besteht darin, dass dem Transzendenten die Eigenschaft des Unbeschreiblichen zukommen kann. In diesem Fall entstehen eine Vielfalt indirekter Kommunikationsformen wie Metaphern oder Gleichnisse oder auch Kommunikationsverbote (was z.B. auch Bilderverbote einschließen kann). Zusammen mit der Weitergabe der aus der Kommunikation mit dem Transzendenten entstandenen Kommunikate, die häufig über Jahrhunderte 
oral und/oder schriftlich tradiert und für die jeweilige Lebenssituation immer wieder neu ausgelegt werden, entstehen komplexe, multimodale Kommunikationsformen. Dieses kann noch dadurch gesteigert werden, dass aus den meisten Formen religiöser Praxis eine elaborierte Metaphysik erwächst, deren Wahrheitsanspruch zumeist (und über lange Zeit) über dem anderer Institutionen im politischen und rechtlichen Bereich angesiedelt wird. Bei einer einflussreichen Religion kommt es weiter zu starken Wechselwirkungen mit der jeweiligen Kultur und Sprache. Soziolinguistisch betrachtet stehen wir daher komplexen Glaubens- und Kulturgemeinschaften gegenüber (Bouma/Aarons 2004). Damit können auch die üblichen soziolinguistischen Kategorien auf diese Gemeinschaften angewandt werden. Unter welchen Bedingungen Personen in eine Glaubensgemeinschaft ein- und austreten können, wie sie sich dort im Spannungsfeld von Normierung und Veränderung bewegen, und in welcher Weise das transzendente Sinnbedürfnis gestillt wird, sind einige der Fragen vor allem die Inklusion und Exklusion betreffend, die sich im Blick auf Gemeinschaft ergeben können. Glaubensgemeinschaften können so aber auch nach den spezifischen Kommunikationsformen, konkreter nach der Ausprägung einer besonderen Sprache im Sinne einer Varietät, befragt werden.

Was wir aus dieser kurzen Analyse des Feldes gewonnen haben, sind folgende Begriffe: das Transzendente, die Figur des (charismatischen) Vermittlers, eine religionsspezifische Metaphysik sowie soziale Gruppen und ihre Eigenschaften wie etwa die Mitgliedschaft in einer Glaubensgemeinschaft. Linguistisch kann das kommunikative Verhältnis dieser Figuren interessant werden, bspw. widmet sich Lasch (2011) der Verkündigung, der Verehrung und Vergegenwärtigung kommunikationstypologisch. Während Verehrung durchaus ein polyvalentes Kommunikationsmuster darstellt, das in jedem kulturellen Segment prinzipiell verankert sein kann, ist die Verkündigung für den Bereich der Religion insofern spezifisch, als die Verkündigung eine (charismatische) Mittlerfigur voraussetzt, die Wissen direkt vom Transzendenten erhält und dieses weitergibt - daraus können (aber müssen nicht) Glaubensinhalte entstehen, die dann möglicherweise in einem weiteren Schritt in institutionalisierten Formen der Verkündigung prozessiert werden. Der Inhalt der Verkündigung ist eine nicht anzweifelbare Mitteilung von Sachverhalten und Urteilen über Sachverhalte. Im Bereich der Religion bezeiehen sich diese Kommunikate des Transzendenten und der (um Anerkennung bemühten bzw. anerkannten) Vermittler auf ,eine Welt', in der Transzendenz und Immanenz nicht geschieden sind. Die Aufhebung der Trennung von Immanenz und Transzendenz muss dabei aber nicht ausschließlich über das Wort erfolgen, auch die Performanz kann bspw. neben der Vergegenwärtigung einer Verkündigungssituation selbst Qualitäten der Verkündigung tragen, wenn bspw. anerkannt ist, dass der Vermittler während der Performanz in Kontakt mit dem Transzendenten steht. Die Rolle des Gläubigen verlangt dabei, dass dieser das Verkündigte als vom Transzendenten stammende Botschaft akzeptiert und - je nach Tradition - in Form einer Auslegung als alltagsrelevant in seine Ethnomethoden integriert. Strukturanalogien ergeben sich hier zu den Bereichen des Rechts und des Politischen (die 
schlussendlich aus dem des Religiösen erwachsen sind), nur dass es sich hierbei um eine weltliche Autorität in Form einer Person oder einer Institution handelt - die Existenz des Konzeptes und Begriffs „Ersatzreligion“ deutet an, wie wirkmächtig kommunikative Muster der Verkündigung alle gesellschaftlichen und kulturellen Bereiche (auch und gerade in ,säkularen' Gesellschaften) erfassen können.

Die soziolinguistische Betrachtung der Religionen als Glaubens- und Kulturgemeinschaften lenkt den Blick zudem auf die Umwelt von religiösen Gruppen und Traditionen. Wenn sich Inklusions- und Exklusionsmechanismen beschreiben lassen, muss es Umwelten geben, die nicht Teil des Weltausschnitts sind, den die Glaubensund Kulturgemeinschaft ideologisch für sich reklamiert. Solche Interferenzen sind als linguistischer Gegenstand zu postulieren: Wie kommunizieren (charismatische) Vermittler verschiedener Glaubensgemeinschaften miteinander, wie diejenigen, die der einen oder der anderen Glaubensgemeinschaft angehören? Zwischen diesen findet beständig Kommunikation statt, die man in bestimmte Typen einteilen kann, die ganz wesentlich von der Haltung abhängen, die gegenüber dem Anderen eingenommen wird. Werden Menschen, die nicht zur Gemeinschaft gehören, negativ z. B. als ,Nichtgläubige، - im Sinne von ,Nicht-Rechtgläubige ‘ - charakterisiert? Oder wird eine andere Gruppe, Institution und Tradition, als positive Bereicherung konzeptualisiert? Wie verhält man sich gegenüber jenen, die keiner spezifischen Gemeinschaft zuordenbar sind? Diese kommunikativen Kontakte stellen insbesondere für die zukünftigen Forschungen ein hochinteressantes Feld dar, da sich im Kontakt bzw. im imaginierten Kontakt ein großes Potenzial an Konflikten offenbart. Schließlich spielen dabei nicht nur kulturelle, soziale und machtpolitische Faktoren eine Rolle, sondern durchaus auch religionsspezifische (vgl. Assmann 2006) - wie bspw. Reformation und Gegenreformation offenkundig zeigen.

\section{Zentrale Lemmata}

Im Folgenden sollen zentrale Lemmata, die in der situativen Analyse des Feldes „Sprache und Religion“ schlaglichtartig angesprochen wurden, etwas detaillierter erfasst werden. Dass es dabei teils zu Überschneidungen kommen kann und muss, hat seinen Grund darin, dass die Konzepte und Begriffe, auf die hier abgehoben wird, systematisch nicht auf einer Ebene liegen, sondern einzelne Aspekte eines Phänomens, das für die Erforschung des Phänomenbereichs „Sprache und Religion“ von herausragender Bedeutung ist, in den Fokus rücken.

\section{Verkündigung}

Wer verkündigt, reklamiert das Rederecht für sich mit einer ungeheuren Botschaft: Nicht nur das, was gesagt wird, sondern auch, wie es gesagt wird, ist übermenschlich, nicht aus dieser Welt. Menschen, die mit einem solchen Anspruch in Erscheinung 
treten, werden Propheten genannt. Damit sind nicht nur die Propheten des Alten Testaments gemeint. Die Weitung des Begriffs des Prophetischen ist ein großes Verdienst der Religionssoziologie, hier exemplarisch bei Bousset (weiterentwickelt dann bei Weber 1922/1980, 275), zu Beginn des letzten Jahrhunderts (vgl. Lang 2001, 170):

Was den Propheten offenbar geworden ist, und was sie verkünden, das ist eine einheitliche, in sich geschlossene Überzeugung vom Inhalt und Wesen des Lebens, seinen tiefsten Fundamenten und seinen höchsten Zielen. Es ist ein wirklich einheitliches Ganzes, eine geschlossene Überzeugung, die in wenigen Sätzen zusammengefasst werden kann, nicht mehr ein buntes Vielerlei von Gewohnheit, Herkommen, Volkssitten, kultischen und rituellen Forderungen, ekstatischen Äußerungen, moralischen Sätzen. (Bousset 1903, 107)

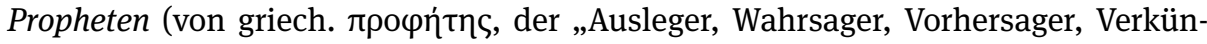
der“; vgl. Klein 1997, 475) sind göttlich inspiriert, Träger des Charismas als einer „von Gott dauerhaft verliehene[n] Gabe“ (Lang 2001, 172) und stellen als Träger des persönlichen Charismas das Amtscharisma der Priester eines/r etablierten Kultes/Religion in Frage (Klein 1997, 476; zum Charisma vgl. die Lemmabeschreibung unten). Solche „ethischen Propheten (Sendungspropheten)“ haben Teil an der göttlichen Offenbarung, die sie mittels komplexer Zeichensysteme in die Kommunikationswelt der Menschen transferieren - sie haben als Träger göttlichen Charismas zugleich alleinige Auslegungsmacht über das offenbarte Wissen und die dafür verwendeten komplexen Symbole. „Exemplarische Propheten“, wie z. B. Buddha (im Anschluss an Weber vgl. Lang 2001, 173f.), unterscheiden sich nur insofern von „ethischen Propheten“, als dass sie nicht selbst postulieren, Träger göttlichen Charismas und Wissens zu sein (und daher auch nicht Gottes Wort bringen), sondern eine Gemeinschaft ihnen diese Qualität zuweist und deshalb nachfolgt: Charisma konstituiert sich über Anhänger. Die Zeichen des Propheten werden - etabliert sich ein neuer Kult - von Priestern in der Nachfolge verwaltet (Lang 2001, 176). Priester üben sich, institutionell abgesichert, in die nachprophetische Verkündigung und Auslegung ein - als Träger des Amtscharismas organisieren sie stabilisierendes rituelles Handeln; sie stellen das einmalige und singuläre prophetische Ereignis auf Dauer. Allerdings beschränkt sich die Aufgabe der „nachprophetischen Gestalten“ (Lang 2001, 175) nicht darauf, sondern sie wirken aktiv an der Ausgestaltung der Glaubensinhalte mit - neben religiöse Praxen tritt eine Lehre, eine Theologie, in der das Wissen um religiöse Symbole und Glaubenssätze systematisiert wird (vgl. Weber 1922/1980). Ihre wichtigste Handlung ist jedoch die der Verkündigung. Hier sprechen sie, wie der (ethische) Prophet, in der Sprache der Menschen Gottes Wort. Oder sie stellen, z. B. durch Imitation, die göttliche Inspiration eines (exemplarischen) Propheten aus. In beiden Fällen bringen sie Botschaft einer Gottheit und vergegenwärtigen zugleich das prophetische Ereignis. Die Verkündigung ist in diesem Sinne das kommunikative Muster, welches religiöse Kommunikation von anderen maßgeblich unterscheidet - göttliches, prinzipiell durch den Menschen nicht verfügbares, Wort und Wissen wird durch menschliches Wort und Handeln zur Anschauung gebracht (vgl. Dembowski 2002, 714); die Samm- 
lung und Archivierung dieses Wortes und Wissens (etwa in Heiligen Texten) ist dem nachgelagert. Die Verkündigung ist monologische Transferleistung auf einer vertikalen kommunikativen Achse; anschließende kommunikative Handlungen wie etwa Interpretationsleistungen sind notwendigerweise auf einer horizontalen kommunikativen Achse anzusiedeln.

\section{Verehrung}

Die zentralen kommunikativen Formen der Verehrung sind das Bekenntnis und das Gebet, (Opfer-)Gaben oder Unterwerfungshandlungen, Gesang, rituelle Tänze und andere Arrangements der Körper der Gläubigen usw.: In der Verehrung tritt die Multimodalität religiöser Kommunikation offen zu Tage. Die Verehrung ist „eine heilige Handlung, die in rituell feststehenden Formen von einer Gemeinschaft vollzogen wird, in deren Vertretung ein oder mehrere Zelebranten die gottesdienstlichen Akte vollziehen“ (Lanczkowksi 1993, 1) - ist darauf aber, z. B. in nicht institutionalisierten persönlichen Religionen, freilich nicht beschränkt. Verehrungsakte sind kommunikativ - wie die Verkündigung - primär vertikal organisiert, haben allerdings durch die rituelle Einbettung als kommunikative Akte starke Wirkung auf die Kultgemeinschaft, da sie die Aufmerksamkeit und die kommunikativen, gemeinschaftlichen Handlungen auf einen gemeinsamen Fokus ausrichten. Die vertikale Kommunikation - sei damit das Transzendente bzw. ein Mittler adressiert - wird in den meisten Fällen monologisch bleiben. Den Verwaltern religiösen Wissens kommt die Aufgabe zu, die Zeichen zur Interpretation des Gotteswillens heranzuziehen und darauf mit rituellen Handlungen zu reagieren, die ,in der einen Wirklichkeit * in der Transzendenz und Immanenz nicht analytisch geschieden sind - auch reale Wirkungen verursachen. Diese Verwalter oder Priester verfügen - im Gegensatz zum singulären Handeln des Propheten - über ein in der Tradition systematisiertes (Handlungs-)Wissen, um die Verantwortung für positive und negative Folgen der kommunikativen Akte der Verehrung auf das (Fehl-)Verhalten der Kult- oder Glaubensgemeinschaft zu verlagern (Weber 1922/1980, 261).

\section{Vergegenwärtigung}

Verkündigung und Verehrung weisen - religionsgeschichtlich betrachtet (Lanczkowksi 1993, 2) - nicht nur in Bezug auf ihre Ordnung und ihre als adäquat bewerteten kommunikativen Handlungen eine außerordentliche Stabilität auf, sondern auch in Bezug auf die Zeiten und Räume, an denen sie vollzogen werden. Diese unterliegen einer Eigengesetzlichkeit: Kommunikative Handlungen, Heterotopien und Heterochronien (vgl. Foucault 2005) sind unlösbar miteinander verbunden, bilden multimedial und multikodal den Symbolhaushalt religiöser Praxen ab und bringen ihn gleichzeitig immer wieder hervor. Heterotopien sind die Plätze der Verkündigung und Verehrung, die je nach Religionsgemeinschaft unterschiedlich ausgestaltet sind. Rituelle Handlungen dienen der Vergegenwärtigung und machen diese Orte der Eigengesetzlichkeit auch zu Orten der Eigenzeitlichkeit: Rituale sind zeitlich unmittelbar, gestalten 
aber die Gegenwart durch die Vergegenwärtigung des Vergangenen (vgl. zu diesem Komplex u. a. Turner 1989, 1967; Soeffner 1989; Werlen 1984; Paul 1990; Fix 1998; Kauke 1998; Belliger/Krieger 1998; Rappaport 1998 und Lasch 2005, 2011). Verkündigung und Verehrung sind an solche anderen (heiligen) Orte und Zeiten gebunden und in Riten eingebettet. Das hat auch Konsequenzen für das zentrale Symbolsystem, die Sprache, die durch ihre Verwendung im Ritual in einem spezifischen Zusammenhang funktionalisiert, um nicht zu sagen, sakralisiert wird.

Rituale dienen aber nicht nur allein der Vergegenwärtigung des (vergangenen singulären) Verkündigungsereignisses, sondern sie machen zugleich das Transzendente in der Menschenwelt (spürbar) gegenwärtig: Durch (persönliches oder institutionalisiertes) Ritual an spezifischen Plätzen und zu besonderen Zeiten und durch die Nutzung bestimmter Gegenstände wird das Transzendente präsent.

\section{Charisma}

Charisma - verstanden im Weber'schen Sinne - stellt eine zentrale Kategorie für den Bereich „Sprache und Religion“ dar, da der Träger von Charisma eine direkte Verbindung zum Transzendenten aufweisen soll, die diesen vor anderen heraushebt und ihm damit eine Führungsrolle innerhalb einer Gemeinschaft von Anhängern zuspricht. Max Weber ordnete dem Begriff des Charismas den Beweis durch Wundertaten - einen Akt der Verkündigung - untrennbar zu. Viel mehr als über den Träger des Charismas kann man über die Gemeinschaft in Erfahrung bringen, die seine Wundertaten bewertet - denn was ein Wunder sei, ist kollektiv bestimmt. War z. B. eine spontane Heilung vor 2000 Jahren ein solches unfassbares Wunder, so ist es heute z. B. auch eine als authentisch akzeptierte Gotteserfahrung, die als Präsenz des Transzendenten für Gläubige (einer Gemeinschaft) greifbar wird (vgl. etwa Strohschneider 2009 zur Nacharbeitung dieses Musters in narrativen Entwürfen). Die Teilhabe an dieser Präsenz bei bestimmten Vergemeinschaftungsveranstaltungen oder das Rezipieren von Kommunikaten wäre dann eine Minimalvoraussetzung für das, was Weber den „Erfolg“ oder das „Wohlergehen“ nennt:

Der Träger des Charisma ergreift die ihm angemessene Aufgabe und verlangt Gehorsam und Gefolgschaft kraft seiner Sendung. Ob er sie findet, entscheidet der Erfolg. Erkennen diejenigen, an die er sich gesandt fühlt, seine Sendung nicht an, so bricht sein Anspruch zusammen. Erkennen sie ihn an, so ist er ihr Herr, solange er sich durch „Bewährung“ die Anerkennung zu erhalten weiß. [...] Vor allem aber muß sich seine göttliche Sendung darin ,bewähren', daß es denen, die sich ihm gläubig hingeben, wohlergeht. Wenn nicht, so ist er offenbar nicht der von den Göttern gesandte Herr. (Weber, 1922/1980, 655/656, Herv. i. Orig.)

Charisma definiert bei Weber den Typus der charismatischen Herrschaft, es konstituiert sich im Wesentlichen durch Kommunikation und Handlungen zwischen dem Träger des Charismas und seinen Anhängern. Das Amtscharisma steht in seiner Bedeutung dem nicht nach - Vertreter einer Institution stellen das singuläre prophe- 
tische Ereignis auf Dauer, indem Wissen tradiert, kommuniziert und in stabilisierenden rituellen Handlungen vergegenwärtigt wird (vgl. Kitzinger 2009).

\section{Unsagbarkeit}

Ein zentrales Moment für die Kommunikation religiöser Erfahrung ist die Unsagbarkeit, Unbeschreiblichkeit oder Unaussprechbarkeit, die sich aus unterschiedlichen Erfahrungen speist und das kommunikative Muster des Unsagbarkeitstopos hervorbringt. Dieser Topos erscheint zunächst in Situationen, in denen das Subjekt eine Erfahrung gemacht hat resp. vorgibt, sie gemacht zu haben, die so außergewöhnlich ist, dass es nicht mehr auf die gewohnten Kommunikationsroutinen zurückgreifen kann und dann ,schwelgt', ,stammelt', ,verstummt', ,weint' oder am ganzen Körper ,zittert‘ (Kommerell 1962; Heimböckel 2003; Gülich 2005; Lasch 2005; Schiewer et al. 2010). Eine weitere Quelle für den Unsagbarkeitstopos sind die Phänomene, in denen das Transzendente teilweise oder ganz die Kontrolle über einen Menschen (meist eine Mittlerfigur) übernimmt und dann „durch“ diesen „spricht“ - die Zeichen (wie z. B. das ,Zittern') können dabei ambivalenter Natur sein. Dazu zählen der Pfingstmythos, alle Formen der Besessenheit oder auch schamanistische und okkult-mediale Praktiken und Erfahrungen. Der Unsagbarkeitstopos spielt auch in der Sprachphilosophie eine wichtige Rolle. Wenn Ludwig Wittgenstein etwa schreibt „Es gibt allerdings Unaussprechliches. Dies zeigt sich, es ist das Mystische“ (Wittgenstein 1916/1966, 155, 6.522, Herv. i. Orig.), dann verweist der Ausdruck „Unaussprechliches“ bereits auf etwas, das gar nicht möglich ist. Über das Unaussprechliche zu sprechen konstituiert eine der basalen Paradoxien religiöser Kommunikation, denn wenn etwas unaussprechlich ist, dann kann es eben nicht ausgesprochen werden, auch nicht durch ein Wort wie „Unaussprechliches“ oder wortreichere Umschreibungen. Will man das Unaussprechliche also in irgendeiner Form kommunizieren, bewegt man sich immer in einer paradoxen Situation, in der die Behauptung eines Unaussprechlichen durch ihr bloßes Aussprechen als unhaltbar entlarvt wird. Zum anderen ist diese basale Paradoxie und ihre Ausnutzung im Unsagbarkeitstopos Ausweis und Schutz eines Gläubigen zugleich: Bedient man sich des Topos, sagt man, im übertragenen Sinne, mehr als tausend Worte vor sich, der Gemeinschaft und im Angesicht des Transzendenten.

Eine weitere Quelle des Unsagbarkeitstopos stellt die religiöse Erfahrung eines ,jenseits von Kategorien' dar, die dann mit einer Sinnformel wie der coincidentia oppositorum, d. h. der Aufhebung des logischen Identitätspostulats, etwa bei Cusanus beschrieben wird (von Kues 2002).

Schließlich wird der Unsagbarkeitstopos dann genutzt, wenn aus einer Ganzheitserfahrung, wie sie etwa in der ,Mystik ${ }^{\star}$ bei Meister Eckhart zu finden ist, die paradoxe Situation erwächst, dass ein Subjekt ein Ganzes beschreiben soll, dessen bloßes Teil es ist.

Der Unsagbarkeitstopos findet seinen Ausdruck in einer Reihe indirekter Redeformen. Dazu zählen insbesondere Metaphern, Gleichnisse, aber auch narrativ ent- 
wickelte Paradoxien, wie sie etwa in zen-buddhistischen Koans zu finden sind. Wie Sprache kann auch die Verwendung bzw. der explizite Ausschluss der verfügbaren kommunikativen Codes (z. B. Bilder, Kleidung, körperliche Ausdrucksformen wie Tanz oder Gesang) Ausweis des Unsagbarkeitstopos sein.

\section{Das Transzendente}

Grundlage der Religion ist die Erfahrung einer anderen Sichtweise, die für das betroffene Subjekt mit mehr oder weniger großen physischen, psychischen und sozialen Veränderungen verbunden ist. Zumeist wird diese Erfahrung als eine neue Perspektive, manchmal auch als die eigentliche Perspektive, oder einfach als die „Wahrheit“ bezeichnet. Die perspektivische Sichtweise ist modern und resultiert aus den Bedingtheiten der modernen Gesellschaft, in der nicht mehr ein Deutungsangebot der Welt und Wirklichkeit und Wahrheit das Monopol auf Deutung hat, sondern in der viele Stimmen in einer pluralistischen Gesellschaft konkurrierende Deutungsangebote offerieren. In vormodernen Gesellschaften des christlichen Europa wirkt das Transzendente immer in der Immanenz, es gibt in der Wahrnehmung nur die Welt, in der auch das Transzendente sich immer zeigt und gedeutet werden muss. Dennoch unterscheidet sich das damit einhergehende Gefühl, Teil von etwas Umfassenderem zu sein, nicht, auch wenn seine Voraussetzungen in vormodernen und modernen Gesellschaften je andere sind - aber das Gefühl wird in den verschiedenen Religionen unterschiedlich konzeptualisiert. Dies reicht von der Annahme von Entitäten in polytheistischen, monotheistischen und paganistischen Glaubensrichtungen, bis hin zu monistischen Vorstellungen eines Verlöschens des Ichs im Buddhismus oder im Advaita. Gerade der Buddhismus in seinen zeitgenössischen Spielarten hat aber gezeigt, dass die monistische Auffassung nicht zu einer Aufgabe von transzendenten Wesenheiten oder Energien führen muss, vielmehr besteht auch dort ein elaboriertes System von Buddhas und anderen transzendenten Wesenheiten (vgl. Keown 2010).

Häufig wird das in einer Glaubensgemeinschaft angenommene Transzendente mit strikten Darstellungsnormen und/oder -tabus belegt. Auch dies stellt eine Quelle für verschiedene Formen des indirekten Sprechens (s. o.) dar.

\section{Metaphysik}

Ein zentrales Kennzeichen von Religionen ist eine eigene Metaphysik, die in der Regel als vom Transzendenten stammende Autorität verstanden wird und das menschliche Bedürfnis nach Sinn und Sinnformeln stillt (Geideck/Liebert 2003). In der Spiritualität der Spätmoderne stellt diese Metaphysik häufig eine idiosynkratische Brikolage dar (vgl. Gebhardt et. al. 2005). Diese Metaphysiken sind häufig in kultischen Texten kodiert, denen eine ähnliche Verehrung zu Teil wird wie dem Transzendenten selbst. Aus soziolinguistischer Sicht sind v. a. die Dynamiken einschließlich der Konflikte relevant, die diese Metaphysiken in einer heterogenen Umgebung wie anderen Religionen oder säkularen Gesellschaften entfalten. Außerdem ist die Hermeneutik der Teilnehmer einer religiösen Tradition von Interesse und damit die Frage, wie die 
häufig aus anderen Sprachepochen stammenden Texte für die Gegenwart gedeutet und interpretiert werden. Aber auch auf der performativen Ebene sind diese Metaphysiken relevant, insbesondere wenn sie mit einem politischen Geltungsanspruch in der Gesellschaft auftreten (Kreationismus, Sharia, Jihat).

\section{Methodische Überlegungen}

Die Beobachtung und Beschreibung der Art und Weise der Kommunikation über religös-metaphysische Inhalte stellt den Ausgangspunkt jeglicher methodischen Überlegung dar. Diese Kommunikation kann in institutionalisierten Religionen ebenso wie in informellen und flüchtigen Gemeinschaften wie auch im Bereich der persönlichen Religion stattfinden. Es handelt sich hierbei aber nicht nur um die Kommunikation von Vertretern bestimmter Glaubensgemeinschaften, sondern auch um institutionell nicht gebundene Personen, die Religion im weitesten Sinne thematisieren und insbesondere den spezifisch religionskritischen Diskurs, der sich auf die Aufklärung, den Laizismus oder andere religionskritische Grundlagen beruft.

Inwieweit hier von einer Generierung von Wissen gesprochen werden kann, ist ebenso sehr Gegenstand methodischer Überlegungen wie die Frage der Standortgebundenheit der untersuchten Gemeinschaft und des sie untersuchenden Forschers. Bei letzterer Frage gibt es also durchaus eine Verwandtschaft zur Analyse politischer Sprache.

Wie oben erwähnt, wurden bisher in der Regel lexikologische, lexikographische, textlinguistische und auch editionsphilologische Verfahren verwendet, um sich dem Phänomen „Sprache und Religion“ zu nähern. Auf neuere, multimodale Ansätze wurde hier ebenso hingewiesen wie auf die ethnografischen und soziologischen Verfahren, die in der Linguistik bislang noch nicht eingesetzt wurden. Um kulturell relevante Prozesse der Generierung, Elaboration, Speicherung und Vermittlung religiösmetaphysischer Inhalte in ihren gesellschaftlichen Kontexten $\mathrm{zu}$ beschreiben, bietet sich darüber hinaus zudem die Diskursanalyse an.

Damit sind zunächst die im engeren Sinn an Michel Foucault (1974, 1981 und 2007) angelehnten Ansätze (z. B. Keller 2010) angesprochen. „Diskurs“ ist bei Foucault eine „Menge von Aussagen, die einem gleichen [Wissens-]Formationssystem angehören“ (Foucault 1981, 156). Diese Formationssysteme, so seine Überzeugung, lassen sich aus den „fundamentalen Codes einer Kultur, die ihre Sprache, ihre Wahrnehmungsschemata, ihren Austausch, ihre Techniken, ihre Werte, die Hierarchie ihrer Praktiken beherrschen,“ erschließen. Denn diese „fixieren gleich zu Anfang für jeden Menschen die empirischen Ordnungen, mit denen er zu tun haben und in denen er sich wiederfinden wird“ (Foucault 1974, 22).

Eine linguistische Variante des Foucault'schen Diskursbegriffs ist durch die Historische Semantik Busses (1987) und die Präzisierung des aktualisierten Diskursver- 
ständnisses in einem Forschungsprogramm bei Busse und Teubert (1994, vgl. auch Felder 2006; Felder/Müller 2009) im Sinne von thematisch gebundenen Diskursen vorgenommen worden.

Begreift man die „Sprache der Religion“ soziolinguistisch als funktionale Varietät, nimmt man die hier vorgestellten Lemmata als konstitutiv für die Beschreibung dieser Varietät und bettet diskurslinguistische Untersuchungen $\mathrm{zu}$ „Sprache und Religion“ in eine solche Soziolinguistik ein (vgl. auch Lasch 2013), wird auch der Begriff der Macht, den Foucault in seinen späteren Arbeiten beinahe dominant setzte, in Zukunft eine ganz andere Rollen spielen müssen. Sprechen wir über Religionen als Glaubensgemeinschaften, die ideologisch die Durchsetzung einer spezifisch ,richtigen' Sicht auf Wirklichkeit (als ,eine Wahrheit') verfolgen, wird man die Mechanismen beschreiben müssen, die Foucault in der Macht des Diskurses analysierte - es gibt nicht einen Platz der Macht, sondern so viele Plätze, wie es Wissen gibt: Wissen ist nicht Ergebnis, sondern Ausdruck von Macht; mittels der konstruierten und postulierten und verfochtenen Wahrheiten werden Machtverhältnisse erst sichtbar, die gestützt werden sollen und mit anderen konfligieren (zuletzt Foucault 2006; vgl. dazu am Beispiel des Bundes der Assassinen Lasch 2012).

\section{Literaturverzeichnis}

Ammon, Ulrich/Jeroen Darquennes/Sue Wright (Hg.) (2011): Sprache und Religion. Berlin

(Sociolinguistica, 25).

Assmann, Jan (2006): Monotheismus und die Sprache der Gewalt. Vortrag im Alten Rathaus am 17.

November 2004. Wien (Wiener Vorlesungen im Rathaus; 116).

Belliger, Andréa/David J. Krieger (1998): Einführung. In: Andrea Belliger/David J. Krieger (Hg.):

Ritualtheorien. Ein einführendes Handbuch. Wiesbaden, 7-33.

Bouma, Gary D./Haydn Aarons (2004): Art. 42. Religion. In: Ulrich Ammon u. a. (Hg.): Soziolinguistik.

2 Bde. 2. Aufl. Berlin/New York (Handbücher zur Sprach- und Kommunikationswissenschaft,

3.1), 351-354.

Bousset, Wilhelm (1903): Das Wesen der Religion, dargestellt an ihrer Geschichte. Halle/Saale.

Busse, Dietrich (1987): Historische Semantik. Analyse eines Programms. Stuttgart (Sprache und

Geschichte, 13).

Busse, Dietrich/Wolfgang Teubert (1994): Ist „Diskurs“ ein sprachwissenschaftliches Objekt? Zur Methodenfrage der historischen Semantik. In: Dies./Fritz Hermanns (Hg.): Begriffsgeschichte und Diskursgeschichte. Methodenfragen und Forschungsergebnisse der historischen Semantik. Opladen, 10-28. Erscheint erneut in: Dies. (Hg.): Linguistische Diskursanalyse. Wiesbaden.

Dembowski, Hermann (2002): Verkündigung. Dogmatisch. In: Theologische Realenzyklopädie 34, 714-717.

Felder, Ekkehard (2008): Forschungsnetzwerk „Sprache und Wissen“. Zielsetzung und Inhalte. In: Zeitschrift für Germanistische Linguistik ZGL 36(2), 270-276.

Felder, Ekkehard (2009): Wissen durch Sprache. Theorie, Praxis und Erkenntnisinteresse des Forschungsnetzwerkes ,Sprache und Wissen'. Berlin/New York (Sprache und Wissen, 3).

Felder, Ekkehard (Hg.) (2006): Semantische Kämpfe. Macht und Sprache in den Wissenschaften. Berlin/New York. 
Felder, Ekkehard/Marcus Müller (Hg.) (2009): Wissen durch Sprache. Theorie, Praxis und Erkenntnisinteresse des Forschungsnetzwerks „Sprache und Wissen“. Berlin/New York (Sprache und Wissen, 3).

Fix, Ulla (1998): Ritualität im Wandel - Projektbericht. In: Ulla Fix (Hg.): Ritualität in der Kommunikation der DDR. Ergänzt durch eine Bibliographie zur Ritualität. Frankfurt a. M., IX-XXII.

Foucault, Michel (1974). Die Ordnung der Dinge. Eine Archäologie der Humanwissenschaften. Übers. des franz. Originals von 1966. Frankfurt a. M.

Foucault, Michel (1981): Archäologie des Wissens. Übers. des franz. Originals von 1969. Frankfurt a.M.

Foucault, Michel (2005): Die Heterotopien - Der utopische Körper. Zwei Radiovorträge. Frankfurt a. M.

Foucault, Michel (2006): Geschichte der Gouvernementalität. 2 Bde. Frankfurt a. M.

Foucault, Michel (2007): Die Ordnung des Diskurses. Inauguralvorlesung am Collège de france. 2. Dezember 1970. 10. Aufl. Frankfurt a. M.

Funk, Tobias (1991): Sprache der Verkündigung in den Konfessionen. Tendenzen religiöser Sprache und konfessionsspezifischer Varianten in den deutschsprachigen Predigten der Gegenwart. Frankfurt a. M.

Gebhardt, Winfried/Martin Engelbrecht/Christoph Bochinger (2005): Die Selbstermächtigung des religiösen Subjekts. Der „spirituelle Wanderer“ als Idealtypus spätmoderner Religiosität. In: Zeitschrift für Religionswissenschaft 13, 133-151.

Geideck, Susan/Wolf-Andreas Liebert (2003): Sinnformeln. Eine soziologisch-linguistische Skizze. In: Dies. (Hg.): Sinnformeln. Linguistische und soziologische Analysen von Leitbildern, Metaphern und anderen kollektiven Orientierungsmustern Berlin/New York, 3-14.

Greule, Albrecht (1992): Über den Beitrag der Sprachwissenschaft zur Kirchenliedforschung. Drei mögliche Zugriffe. In: Zeitschrift für deutsche Philologie 111, 65-77.

Greule, Albrecht (1999a): Die Sprache im Neuen geistlichen Lied. In: Hermann Kurzke/Hermann Ühlein (Hg.): Kirchenlied interdisziplinär. Frankfurt a. M., 83-98.

Greule, Albrecht (1999b): So sie's nicht verstehen, so sollten sie’s nicht singen? Über den Beitrag der Sprachwissenschaft zur Kirchenliedforschung. In: Hermann Kurzke/Hermann Ühlein (Hg.): Kirchenlied interdisziplinär. Frankfurt a. M., 47-64.

Greule, Albrecht (2004): Gesangbuch und Kirchenlied im Textsortenspektrum des Frühneuhochdeutschen. In: Franz Simmler (Hg.): Textsortentypologien und Textallianzen von der Mitte des 15. bis zur Mitte des 16. Jahrhunderts. Berlin, 521-533.

Grözinger, Albrecht (2009): Rhetorik und Stilistik in der Theologie. In: Ulla Fix/Andreas Gardt/ Joachim Knape (Hg.): Rhetorik und Stilistik. Ein internationales Handbuch zeitgenössischer Forschung. Rhetorik und Stilistik, Halbbd. 2. Berlin/New York (Handbücher zur Sprach- und Kommunikationswissenschaft, 31), 1798-1810.

Gülich, Elisabeth (2005): Unbeschreibbarkeit: Rhetorischer Topos - Gattungsmerkmal Formulierungsressource. In: Gesprächsforschung - Online-Zeitschrift zur verbalen Interaktion 6, 222-244, URL: http://www.gespraechsforschung-ozs.de/heft2005/ga-guelich. pdf, Stand: 25.4.2014.

Hausendorf, Heiko/Reinhold Schmitt (2010): Opening up Openings. Zur Struktur der Eröffnungsphase eines Gottesdienstes. In: Lorenza Monada/Reinhold Schmitt (Hg.): Situationseröffnungen. Zur multimodalen Herstellung fokussierter Interaktion. Tübingen (Studien zur Deutschen Sprache, 47), 53-102.

Heimböckel, Dieter (2003): Emphatische Unaussprechlichkeit: Sprachkritik im Werk Heinrich von Kleists. Ein Beitrag zur literarischen Sprachskepsistradition der Moderne. Göttingen (Dienst Am Wort). 
James, William (1997): Die Vielfalt religiöser Erfahrung. Eine Studie über die menschliche Natur. Frankfurt a. M. (Insel Taschenbuch, 1784).

Kaempfert, Manfred (1971): Säkularisation und neue Heiligkeit. Religiöse und religionsbezogene Sprache bei Friedrich Nietzsche. Berlin (Philologische Studien und Quellen, 61).

Kaempfert, Manfred (Hg.) (1983): Probleme der religiösen Sprache. Darmstadt (Wege der Forschung, 442).

Kauke, Wilma (1998): Ritualbeschreibung am Beispiel der Jugendweihe. In: Ulla Fix (Hg.): Ritualität in der Kommunikation der DDR. Ergänzt durch eine Bibliographie zur Ritualität. Frankfurt a. M., 101-214.

Keller, Reiner (2010): Wissenssoziologische Diskursanalyse: Grundlegung eines Forschungsprogramms. 3. Aufl. Wiesbaden (Interdisziplinäre Diskursforschung).

Keown, Damein (2010): Der Buddhismus. 5. Aufl. Stuttgart (Reclams Universal-Bibliothek, 18771). Kitzinger, Martin (2009): De potentia in actum. Mittelalterliches zur Moderne. In: Franz J. Felten/ Annette Kehnel/Stefan Weinfurter ( $\mathrm{Hg}$.): Institution und Charisma. Festschrift für Gert Melville zum 65. Geburtstag. Köln/Weimar/Wien, 305-317.

Klein, Wassilois (1997): Propheten/Prophetie. Religionsgeschichtlich. In: Theologische Realenzyklopädie 27, 473-476.

Klug, Nina-Maria (2012): Das konfessionelle Flugblatt. Eine Studie zur historischen Semiotik und Textanalyse. Berlin/Boston (SLG, 112).

Knoblauch, Hubert (1989): Das unsichtbare neue Zeitalter. „New Age“, privatisierte Religion und kultisches Milieu. In: Kölner Zeitschrift für Soziologie und Sozialpsychologie 41(3), 504-525, URL: http://nbn-resolving.de/urn:nbn:de:0168-ssoar-40933, Stand: 26.4.2014.

Knoblauch, Hubert (1999): Religionssoziologie. Berlin/New York (Sammlung Göschen, 2094).

Knoblauch, Hubert (2003): Qualitative Religionsforschung. Religionsethnographie in der eigenen Gesellschaft. Paderborn u. a. (Uni-Taschenbücher, 2409).

Knoblauch, Hubert (2009): Populäre Religion. Auf dem Weg in eine spirituelle Gesellschaft. Frankfurt a. M.

Kommerell, Max (1962): Geist und Buchstabe der Dichtung. Goethe, Schiller, Kleist, Hölderlin. 5. Aufl. Frankfurt a. M.

von Kues, Nicolaus (2002): De docta ignorantia - Die belehrte Unwissenheit. Hamburg (Philosophisch-theologische Werke. Lateinisch - deutsch/Nikolaus von Kues. Mit einer Einl. von Karl Bormann, Teilbd. 1).

Lanczkowski, Günter (1993): Gottesdienst. Religionsgeschichtlich. In: Theologische Realenzyklopädie 14, 1-5.

Lang, Bernhard (2001): Prophet, Priester, Virtuose. In: Hans G. Kippenberg/Martin Riesebrodt (Hg.): Max Webers ,Religionssystematik'. Tübingen, 167-191.

Langen, August (1968): Der Wortschatz des deutschen Pietismus. 2., erg. Aufl. Tübingen.

Lasch, Alexander (2005): Beschreibungen des Lebens in der Zeit. Zur Kommunikation biographischer Texte in den pietistischen Gemeinschaften der Herrnhuter Brüdergemeine und der Dresdner Diakonissenschwesternschaft im 19. Jahrhundert. Münster (Germanistik, 31).

Lasch, Alexander (2011): Texte im Handlungsbereich der Religion. In: Stephan Habscheid (Hg.): Textsorten, Handlungsmuster, Oberflächen. Linguistische Typologien der Kommunikation. Berlin/Boston, 536-555.

Lasch, Alexander (2012): Die A[ssassinen] sollen aus Ägypten stammen - Geschichte(n) eines radikal-islamischen Ordens und ihre Diskursivierung an der Schwelle zur Moderne. In: Christian Braun ( $\mathrm{Hg}$.): Sprache und Geheimnis. Sondersprachenforschung im Spannungsfeld zwischen Arkanem und Profanem. Berlin (Lingua Historica Germanica, 4), 89-106.

Lasch, Alexander (2013): Sind serielle Texte ein Gegenstand linguistischer Diskursanalyse? Zu diskursbestätigenden und diskursverändernden „Lebensbeschreibungen“ in rituellen 
Kontexten. In: Dietrich Busse/Wolfgang Teubert (Hg.): Linguistische Diskursanalyse. Neue Perspektiven. Wiesbaden, 65-95.

Moser, Dietz-Rüdiger (1981): Verkündigung durch Volksgesang. Studien zur Liedpropaganda und -katechese der Gegenreformation. Berlin.

Moser, Hugo (1964): Sprache und Religion. Zur muttersprachlichen Erschließung des religiösen Bereichs. Düsseldorf (Wirkendes Wort, Beiheft 7).

Paul, Ingwer (1990): Rituelle Kommunikation. Sprachliche Verfahren zur Konstitution ritueller Bedeutung und zur Organisation des Rituals. Tübingen.

Paul, Ingwer (2009): Rhetorisch-stilistische Eigenschaften der Sprache von Religion und Kirche. In: Ulla Fix/Andreas Gardt/Joachim Knape (Hg.): Rhetorik und Stilistik. Ein internationales Handbuch zeitgenössischer Forschung. Rhetorik und Stilistik, Halbbd. 2. Berlin/New York (Handbücher zur Sprach- und Kommunikationswissenschaft, 31), 2257-2274.

Pfefferkorn, Oliver (2005a): Predigt und Andacht als Textsorten der protestantischen Erbauungsliteratur des 17. Jahrhunderts. In: Zeitschrift für deutsche Philologie 124, 375-394.

Pfefferkorn, Oliver (2005b): ,Übung der Gottseligkeit'. Die Textsorten Predigt, Andacht und Gebet im deutschen Protestantismus des späten 16. und des 17. Jahrhunderts. Frankfurt a. M. (Deutsche Sprachgeschichte. Texte und Untersuchungen, 1).

von Polenz, Peter (1994): Deutsche Sprachgeschichte vom Mittelalter bis zur Gegenwart. Bd. 1:Einführung, Grundbegriffe, Deutsch in der frühbürgerlichen Zeit. Berlin.

von Polenz, Peter (2000): Deutsche Sprachgeschichte vom Mittelalter bis zur Gegenwart. Bd. 1: Einführung, Grundbegriffe, 14. bis 16. Jahrhundert. 2., überarb. und erg. Aufl. Berlin.

Rappaport, Roy A. (1998): Ritual und performative Sprache. In: Andréa Belliger/David J. Krieger (Hg.): Ritualtheorien. Ein einführendes Handbuch. Wiesbaden, 191-211.

Roelcke, Thorsten (2010): Fachsprachen. 3. Aufl. Berlin (Grundlagen der Germanistik, 37).

Schiewer, Hans-Jochen/Stefan Seeber/Markus Stock (Hg.) (2010): Schmerz in der Literatur des Mittelalters und der Frühen Neuzeit. Göttingen (Transatlantische Studien zu Mittelalter und Früher Neuzeit, 4).

Schmitt, Reinhold (2012): Störung und Reparatur eines religiösen Ritus: Die erloschene Osterkerze. In: Stephan Habscheid/Wolfgang Klein (Hg.), Dinge und Maschinen in der Kommunikation, Stuttgart/Weimar (Zeitschrift für Literaturwissenschaft und Linguistik 168), 62-91.

Simmler, Franz (2000): Textsorten des religiösen und kirchlichen Bereichs. In: Klaus Brinker u. a. (Hg.): Text- und Gesprächslinguistik. Ein internationales Handbuch zeitgenössischer Forschung. Textlinguistik, Halbbd. 1. Berlin/New York (Handbücher zur Sprach- und Kommunikationswissenschaft, 16), 676-690.

Simmler, Franz (2005a): Luthers Evangelienübersetzung und die Entstehung neuer Makrostrukturen im Deutschen. In: Sprachwissenschaft 30, 161-216.

Simmler, Franz (2005b): Biblische Textsorten: ihre Merkmale und Traditionen von der Mitte des 15. bis zur Mitte des 16. Jahrhunderts. In: Daphnis 33, 379-546.

Simmler, Franz (2007): Liturgische Textsorten und Textallianzen. In: Sandra Reimann/Katja Kessel (Hg.): Wissenschaften im Kontakt. Kooperationsfelder der Deutschen Sprachwissenschaft. Festschrift für Albrecht Greule. Tübingen, 451-468.

Soeffner, Hans-Georg (1989): Die Ordnung der Rituale. Die Auslegung des Alltags - Der Alltag der Auslegung. Frankfurt a. M.

Stötzel, Georg (1966): Zum Nominalstil Meister Eckharts. Die syntaktischen Funktionen grammatischer Verbalabstrakta. In: Wirkendes Wort 16, 289-309.

Strohschneider, Peter (2009): Religiöses Charisma und institutionelle Ordnungen in der UrsulaLegende. In: Franz J. Felten/Annette Kehnel/Stefan Weinfurter (Hg.): Institution und Charisma. Festschrift für Gert Melville zum 65. Geburtstag. Köln/Weimar/Wien, 571-588. 
Turner, Viktor W. (1998): Liminalität und Communitas (1967). In: Andréa Belliger/David J. Krieger

(Hg.): Ritualtheorien. Ein einführendes Handbuch. Wiesbaden, 251-262.

Turner, Viktor. W. (1989): Das Ritual. Struktur und Antistruktur. Frankfurt a. M. (Theorie und

Gesellschaft, 10).

Weber, Max (1922/1980): Wirtschaft und Gesellschaft. Grundriß der verstehenden Soziologie.

Besorgt von Johannes Winckelmann. Studienausgabe. 5., rev. Aufl. Tübingen, 654-661. Zit.

nach http://www.zeno.org/nid/20011439874, Stand: 26.4.2014

Werlen, Iwar (1984): Ritual und Sprache. Zum Verhältnis von Sprechen und Handeln in Ritualen.

Tübingen.

Werlen, Iwar (1987): Die ,Logik‘ ritueller Kommunkation. In: Zeitschrift für Literaturwissenschaft und Linguistik 60, 41-81.

Wittgenstein, Ludwig (1918/1966): Tractatus logico-philosophicus. Logisch-philosophische Abhandlung. Frankfurt a. M. (edition suhrkamp, 12). 